\title{
Therapeutic effects of human umbilical cord mesenchymal stem cell on sepsis-associated encephalopathy in mice by regulating PI3K/AKT pathway
}

\author{
Zhe Zhang ${ }^{1}$, Li Wang ${ }^{2}$, Feng Li ${ }^{1}$, Xiangfeng Qian ${ }^{1}$, Zhixing Hong ${ }^{1}$, Longchuan Wu ${ }^{1}$, Yinsheng Jiang ${ }^{1}$, Haiqiang Hu ${ }^{3,4, *}$ \\ ${ }^{1}$ Department of Emergency Medicine, The First People's Hospital of Yuhang District Hangzhou, 311100 Hangzhou, Zhejiang, China \\ ${ }^{2}$ Department of Emergency Medicine, The Second Affiliated Hospital Zhejiang University School of Medicine, 310002 Hangzhou, Zhejiang, China \\ ${ }^{3}$ Department of Cardiovascular Medicine, The First People's Hospital of Yuhang District Hangzhou, 311100 Hangzhou, Zhejiang, China \\ ${ }^{4}$ Department of Cardiovascular Medicine, The Second Clinical Medical College of Zhejiang Chinese Medicine University, 310053 Hangzhou, Zhejiang, China \\ *Correspondence: haiq_hu@163.com (Haiqiang Hu)
}

\section{DOI:10.31083/j.jin2101038}

This is an open access article under the CC BY 4.0 license (https://creativecommons.org/licenses/by/4.0/).

Submitted: 3 June 2021 Revised: 5 August 2021 Accepted: 15 September 2021 Published: 28 January 2022

Sepsis-associated encephalopathy is a common brain diseases, presenting severe diffuse brain dysfunction. The umbilical cord mesenchymal stem cells have been reported to have protective role for treating diseases, while its role in sepsis-associated encephalopathy remained elusive. This brief report investigated the therapeutic effect of umbilical cord mesenchymal stem cells on sepsis-associated encephalopathy in mice model and uncovering the underlying mechanism. The sepsis-associated encephalopathy mice were injected with $3 \mathrm{mg} / \mathrm{kg}$ lipopolysaccharide. An enzyme-linked immunosorbent assay was carried out to determine the production of inflammatory cytokines. Morris water maze test was used to evaluate mice's neurological dysfunction. Cell apoptosis and tissue injury of the cerebral cortex were assessed using terminal deoxynucleotidyl transferasemediated dUTP nick end labeling (TUNEL) assay and HE staining. Evans Blue leakage detection was used to examine the blood-brain barrier integrity. The protein levels were determined using Western blot. Results showed that the productions of inflammatory cytokines including interleukin 6 (IL-6), interleukin-1 $\beta$ (IL-1 $\beta)$, tumor necrosis factor $\alpha$ (TNF- $\alpha$ ), and high mobility group box protein 1 (HMCB1) and activated NF- $\kappa$ B were increased in sepsis-associated encephalopathy mice, which were decreased by umbilical cord mesenchymal stem cells treatment. Besides, umbilical cord mesenchymal stem cells inhibited lipopolysaccharide-induced cell apoptosis and neuron injury of the cerebral cortex in sepsis-associated encephalopathy mice. Moreover, cognitive dysfunction was observed in sepsis-associated encephalopathy mice, which was alleviated by umbilical cord mesenchymal stem cells. Furthermore, umbilical cord mesenchymal stem cells activated $\mathrm{PI} 3 \mathrm{~K} / \mathrm{AKT}$ signaling pathway. In conclusion, umbilical cord mesenchymal stem cells alleviated inflammation, cell apoptosis and neuron injury of the cerebral cortex, and cognitive dysfunction in sepsis-associated encephalopathy animal model in a $\mathrm{PI} 3 \mathrm{~K} / \mathrm{AKT}$ dependent pathway, making them to be a promising therapeutic strategy for treating sepsis-associated encephalopathy.

\section{Keywords}

Sepsis-associated encephalopathy; Umbilical cord mesenchymal stem cells; Cognitive dysfunction; Cerebral cortex; PI3K/AKT pathway

\section{Introduction}

Sepsis-associated encephalopathy (SAE) is a severe sepsisrelated diffuse brain dysfunction without suffering from direct infection in central nervous system [1]. It is one of the most common brain diseases in intensive care unit (ICU), which seriously threatens patients' health [2]. The pathogenesis of the disease is based on the invasion of bacteria, viruses, or other pathogens, causing acute infection outside the central nervous system and systemic response syndrome [3]. The clinical manifestations of SAE mainly include somnolence, coma, and cognitive impairment [3]. Previous studies have revealed that SAE was an independent predictor of death $[3,4]$. At present, the primary therapeutic strategy for SAE is still limited to managing potential infections [5]. Therefore, it is imperative to explore the effective therapeutic strategy for SAE patients.

In recent years, stem cell therapy has been one of the most promising therapeutic strategy for treating neurologic diseases [6]. Various stem cells derived from neural stem cells, mesenchymal stem cells (MSCs), and umbilical cord blood were considered as therapeutic options for disease treatment [6]. Among these stem cells, MSCs possessed multilineage differentiation, self-renewal, proliferation potential, and the small dosage, making it a valuable therapeutic tool in clinic [7]. MSCs can be isolated from dental pulp, peripheral blood, umbilical cord and bone marrow [8]. However, Shetty et al. [7] report that the MSCs from the umbilical cord are dependable sources of an unlimited number of MSCs for regenerative medicine. At present, umbilical cord mesenchymal stem cells (UC-MSCs) have shown therapeutic roles for treating many diseases in animal models. For example, Xi- 
A
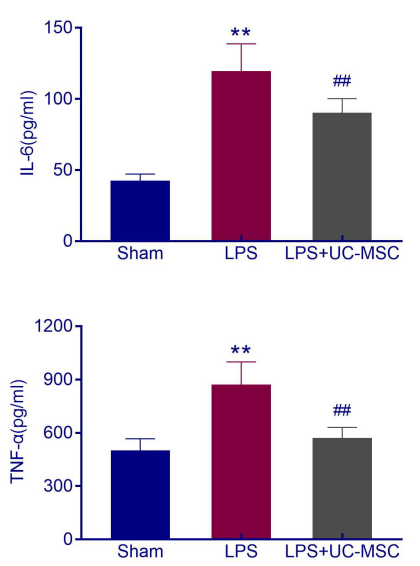

B

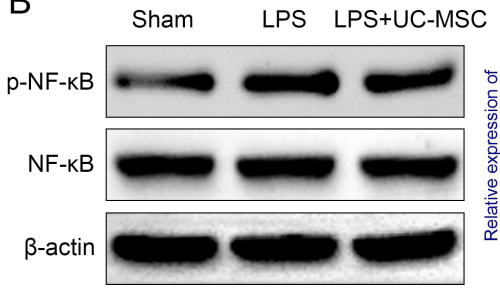

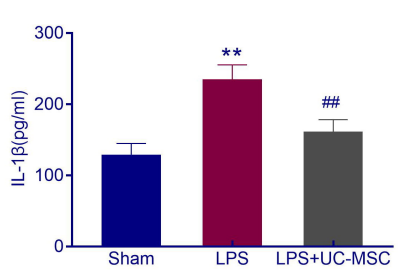
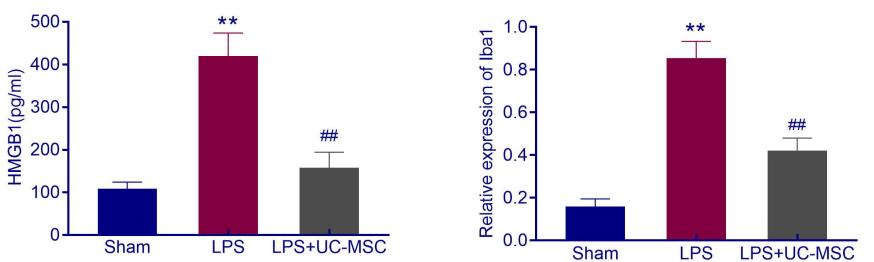

C
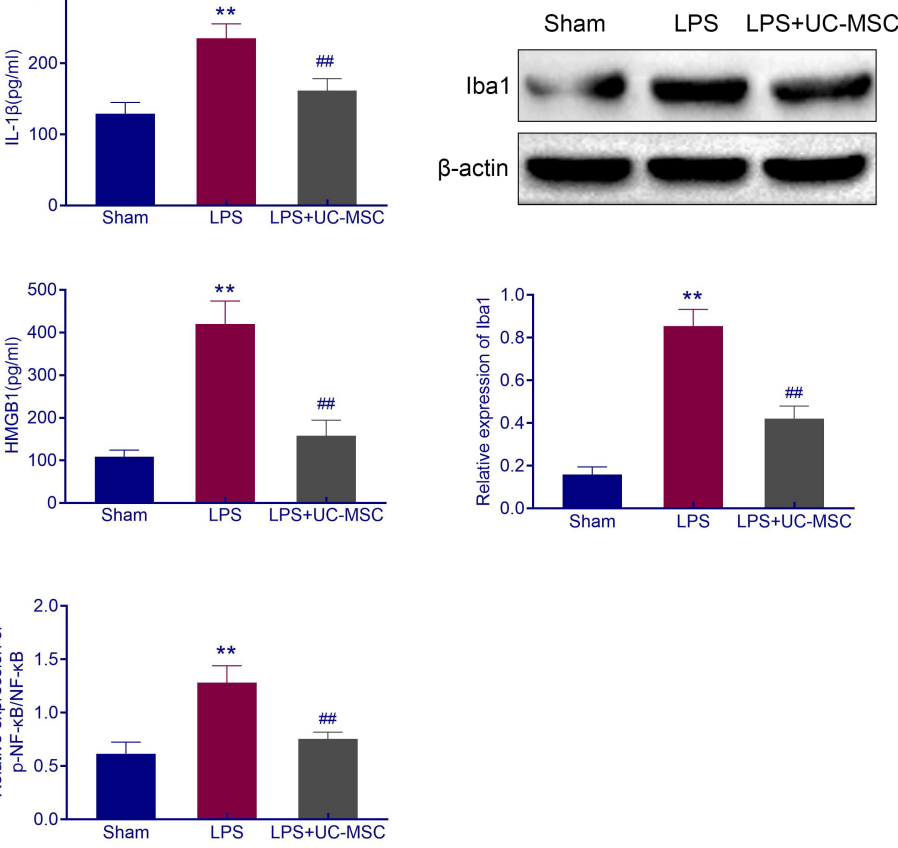

Fig. 1. UC-MSCs inhibited the production of inflammatory factors in SAE mice. (A) The productions of IL-6, IL- $1 \beta$, TNF- $\alpha$, and HMGB1 in brain tissues of UC-MSCs-treated SAE mice were determined using ELISA assay. (B) The protein levels of phosphorylated NF- $\kappa \mathrm{B}$ and total NF- $\kappa \mathrm{B}$ in brain tissues of UC-MSCs-treated SAE mice were detected using Western blot. (C) The protein level of microglia activation related biomarker such as Iba1 in brain tissues of UC-MSCs-treated SAE mice was determined using Western blot. ${ }^{* *}: p<0.01$ means significant difference vs. sham group. \#\#: $p<0.01$ means significant difference vs. LPS group.

ang et al. [9] proved that UC-MSCs can inhibit inflammation and renal fibrosis, resulting in suppressing the development of early diabetic nephropathy. Liu et al. [10] found that UC-MSCs improved the joint damage and osteogenesis in collagen-induced arthritic mice by suppressing TNF- $\alpha$. Thomi et al. [11] demonstrated that UC-MSCs derived from exosomes suppressed the neuroinflammation induced by microglia in perinatal brain injury.

Besides, UC-MSCs have exhibited protective effects on sepsis-related diseases. Zhang et al. [12] reported that human UC-MSCs exosomes could attenuate sepsis-associated acute kidney injury through modulating miR-146b expression. A phase 1 clinical trial of UC-MSCs for treatment of severe sepsis showed that UC-MSCs was safe and well-tolerated and had an excellent therapeutic effect on patients without adverse reactions in 15 patients [13]. However, the effect of UC-MSCs on SAE remained elusive. Hence, we investigate the role of UC-MSCs in the SAE mice and explore the underlying mechanism.

\section{Materials and methods 2.1 Cell culture}

Human UC-MSCs were purchased from ATCC (American Type Culture Collection, Manassas, VA, USA). UCMSCs were cultured with Mesenchymal Stem Cell Basal Media containing growth factors (Mesenchymal Stem Cell
Growth Kit, American Type Culture Collection, Manassas, VA, USA).

\subsection{Animal experiment}

Eighteen male C57BL/6 mice aged 4-6 weeks were acquired from Beijing Laboratory Animal Research Center (Beijing, China). All mice were divided into three groups (6 mice in each group): sham group, LPS group, and LPS + UCMSC group. To determine the effect of UC-MSCs on SAE, the SAE model was first established by injecting mice with lipopolysaccharide (LPS). Except for the mice in the sham group, $3 \mathrm{mg} / \mathrm{kg}$ LPS were injected into all mice intraperitoneally to induce SAE [4]. After $1 \mathrm{~h}$ post LPS injection, the mice in the LPS + UC-MSC group were injected with UCMSCs $\left(1 \times 10^{5}\right.$ cells $)$ using a $35-G$ needle slowly administered over $1 \mathrm{~min}$ via the femoral vein. Eight days later, the cognitive function of mice was evaluated by the Morris water maze test. Mice were euthanized, and brain samples were collected after evaluation of cognitive function was finished. All protocols were conducted according to the guidelines for the care and use of laboratory animals. The Zhejiang Chinese Medicine University permitted this research.

\subsection{ELISA}

The secretion of inflammatory cytokines in SAE mice's brain tissues after administrating with UC-MSCs was determined by ELISA assay. After euthanasia of mice, brain 
tissues of mice were collected to measure the production of interleukin 6 (IL-6), tumor necrosis factor- $\alpha$ (TNF- $\alpha$ ), interleukin 1 beta (IL-1 $\beta$ ) and high mobility group box 1 (HMGB1) using corresponding mouse ELISA kits following the protocols of the manufacturers. All ELISA kits were provided by R\&D Systems (R\&D Systems, Inc., Minneapolis, MN, USA) except for the HMGB1 ELISA kit (Biocompare, California, USA). For detection of IL- 6 , IL- $1 \beta$ and TNF- $\alpha$, $50 \mu \mathrm{L}$ of Assay Diluent RD1N and $50 \mu \mathrm{L}$ of each sample or standard were mixed into the wells of a microplate, and incubated for $2 \mathrm{~h}$ at room temperature. Then, the wells were rinsed with Wash Buffer for 5 times, and $100 \mu \mathrm{L}$ of Mouse IL-6, IL- $1 \beta$ or TNF- $\alpha$ Conjugate were added to each well, incubated for $2 \mathrm{~h}$. The wells were washed again for 5 times and each well was incubated in $100 \mu \mathrm{L}$ of Substrate Solution for $30 \mathrm{~min}$ at room temperature in the dark condition. Finally, of the reaction was terminated by Stop Solution. The optical density of each well measured using microplate reader at $450 \mathrm{~nm}$. For detection of HMGB1, $50 \mu \mathrm{L}$ of each sample or standard were added into the wells of a microplate and incubated for $1 \mathrm{~h}$ at $37^{\circ} \mathrm{C}$. After aspiration, $100 \mu \mathrm{L}$ of Detection Reagent $\mathrm{A}$ was added to the wells and incubated for $1 \mathrm{~h}$ at $37^{\circ} \mathrm{C}$. The wells were then washed for 3 times and $100 \mu \mathrm{L}$ Detection Reagent B was added to the wells with incubation for $1 \mathrm{~h}$ at $37^{\circ} \mathrm{C}$. After that, the wells were washed again for 5 times. Subsequently, $90 \mu \mathrm{L}$ Substrate Solution was added to the wells and incubated for $20 \mathrm{~min}$ at $37^{\circ} \mathrm{C}$, and $50 \mu \mathrm{L}$ Stop Solution was added to the wells. The optical density of each well was measured using microplate reader at $450 \mathrm{~nm}$.

\subsection{Western Blot}

Brain tissue cell lysate was isolated with RIPA lysis reagent (Beyotime Biotechnology, Shanghai, China). The lysate concentration was quantified by the BCA kit (Beyotime Biotechnology, Shanghai, China). The lysate was then subjected to SDS-PAGE and transferred to the PVDF membrane. Subsequently, the PVDF membranes were blocked with 5\% nonfat milk and probed with primary antibodies including antip-NF- $\kappa \mathrm{B}(1: 500), \mathrm{NF}-\kappa \mathrm{B}(1: 1000)$, Iba1 (1:1000), Cleaved caspase-3 (1:500), Bax (1:1000), BCL-2 (1:1000), p-PI3K (1:2000), PI3K (1:1000), p-AKT (1:1000), AKT (1:500) and $\beta$-actin (1:5000) antibodies (Abcam, Cambridge, MA, UK) overnight at $4{ }^{\circ} \mathrm{C}$. The membranes were then incubated with secondary antibody IgG H\&L (HRP) (Abcam, Cambridge, $\mathrm{MA}, \mathrm{UK}$ ) for $1 \mathrm{~h}$ at room temperature. Protein bands were shown utilizing Amersham ECL Prime Western Blotting Detection Reagent (GE Healthcare, Piscataway, NJ, USA). The bands were visualized using ChemiDoc Touch Imaging System (Bio-Rad, Hercules, CA, USA) and quantified using Image $\mathrm{J}$ software (National Institutes of Health, Bethesda, MD, USA). $\beta$-actin was designated as the control protein.

\subsection{TUNEL assay}

Cell apoptosis of cerebral cortex in SAE mice after administration with UC-MSCs was detected using TUNEL assay. The Click-i ${ }^{\mathrm{TM}}$ Plus TUNEL Assay (Thermo Fisher Scien- tific, Waltham, MA, USA) was applied to label fragmented DNA in the nucleus following the manufacturer's protocol. DAPI was used to stain the nuclei of cells, which were then were observed and photographed using a fluorescence microscope (Carl Zeiss Inc., Jena, Germany).

\subsection{Morris water maze test}

Morris water maze test was carried out to evaluate mice's neurological dysfunction as previously described [14-16]. The water maze consisted of a circular black pool $(100 \mathrm{~cm}$ diameter, $38 \mathrm{~cm}$ deep), and it was filled with opaque water ( $25 \mathrm{~cm}$ deep). The water was prepared as black through adding non-toxic pigment, and the temperature of the water was kept at $23 \pm 1{ }^{\circ} \mathrm{C}$. Then, a submerged $(1.5 \mathrm{~cm}$ beneath the water surface) platform was placed into one pool quadrant. All mice underwent three training sessions for 5 days and a probe trial on the sixth day. All mice received four trials with a 15-min interval during each session. To perform the Morris water maze test, the mice were placed in the water facing the wall. All mice possessed 60 seconds to arrive at the platform. The mice were guided to the platform and stayed for $15 \mathrm{~s}$. During the test, the latency to find the platform, the times of mice crossing the platform and swimming speeds were recorded.

\subsection{HE Staining}

HE staining was conducted to assess cerebral cortex neuron injury in SAE mice after administration of UC-MSCs. After euthanasia of mice, brain tissues of mice were collected to detect neuron damage of the cerebral cortex. Tissue samples were fixed in $10 \%$ formalin and embedded into paraffin. Subsequently, $4 \mu \mathrm{m}$-thickness sections were prepared. After deparaffinization and hydration, the sections were stained with hematoxylin and eosin for 5 and 3 minutes, respectively. The staining sections were observed under a light microscope (Carl Zeiss Inc., Jena, Germany).

\subsection{Evans blue leakage detection}

To evaluate blood-brain barrier (BBB) integrity, mice in the different groups were collected to conduct Evans Blue staining. After mice were anesthetized, 2\% Evans Blue (3 $\mathrm{mL} / \mathrm{kg}$ ) in sterile saline solution was injected into the tail vein of mice. After $1 \mathrm{~h}$ circulation, mice were transcardially perfused with cold saline. After that, mice were sacrificed, and the brain tissues were removed and weighed. The tissues were then prepared as homogenate, which was centrifuged for $20 \mathrm{~min}$ at $10000 \mathrm{~g}$. After centrifugation, the supernatant was collected and the absorbance was determined at $620 \mathrm{~nm}$.

\subsection{Statistical analysis}

All data were presented as mean \pm standard deviation (SD), and data was analyzed by SPSS Statistics 22.0 (SPSS, Chicago, IL, USA). The differences among different groups were determined using one-way ANOVA. $p<0.05$ indicated a statistically significant difference. 
A

Sham
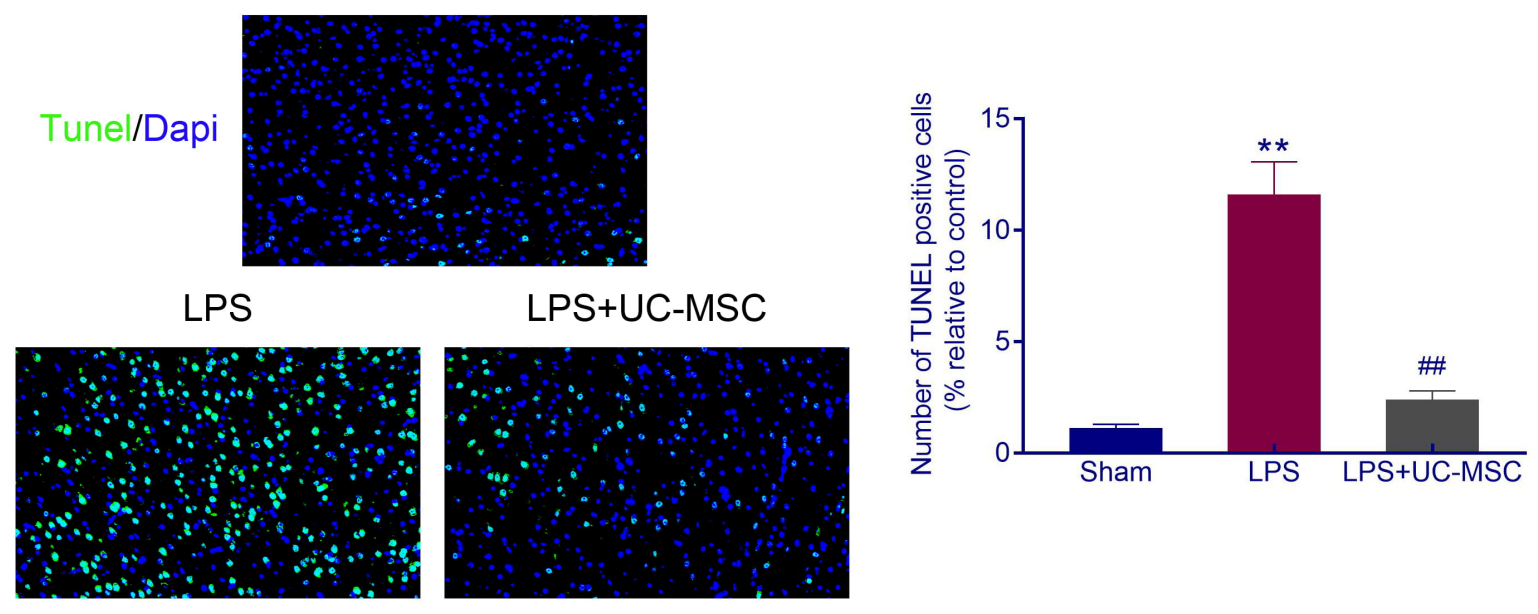

B

Sham LPS LPS+UC-MSC
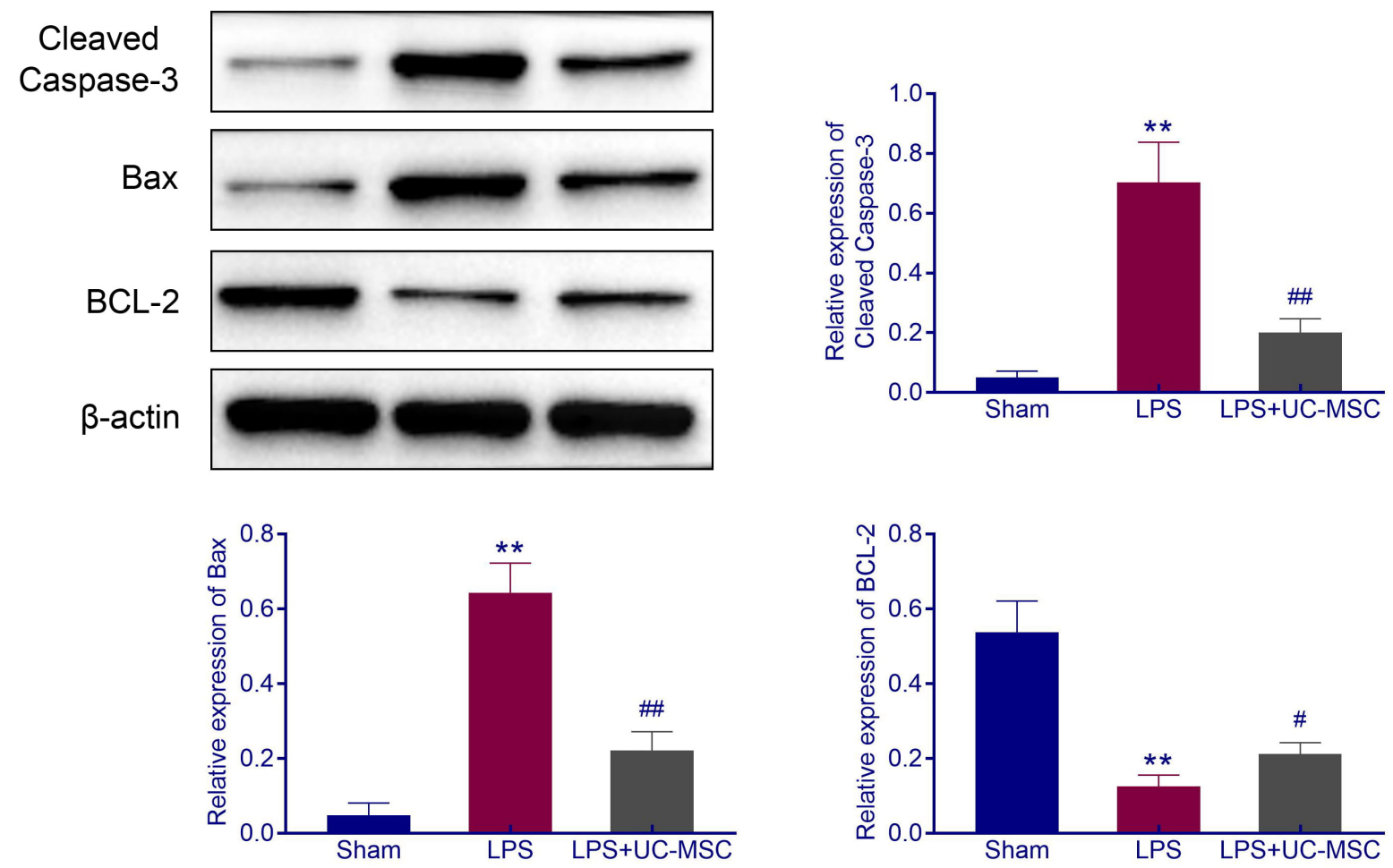

Fig. 2. UC-MSCs inhibited cell apoptosis of the cerebral cortex in SAE mice. (A) Cell apoptosis of cerebral cortex in UC-MSCs-treated SAE mice was evaluated using TUNEL assay. (B) The protein levels of cleaved caspase-3, Bax, and Bcl-2 were detected by Western blot. ${ }^{* *}: p<0.01$ means significant difference vs. sham group. \#: $p<0.05$ means significant difference vs. LPS group. \#\#: $p<0.01$ means significant difference vs. LPS group.

\section{Results}

3.1 UC-MSCs inhibited the production of inflammatory cytokines in SAE mice

The production of IL-6, IL-1 $\beta$, TNF- $\alpha$, and HMGB1 were significantly increased in brain tissues of SAE mice, while which was decreased by UC-MSCs ( $p<0.01$, Fig. 1A). Be- sides, UC-MSCs also inhibited the LPS-induced expression of phosphorylated NF- $\kappa \mathrm{B}(p<0.01$, Fig. 1B). Furthermore, UC-MSCs suppressed the increase of microglia activation related biomarker Iba1 expression in brain tissues of SAE mice $(p<0.01$, Fig. 1C). 
A

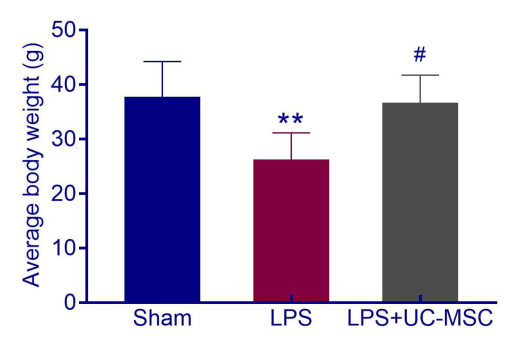

D

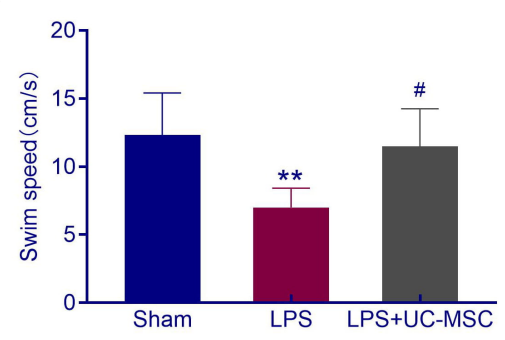

B

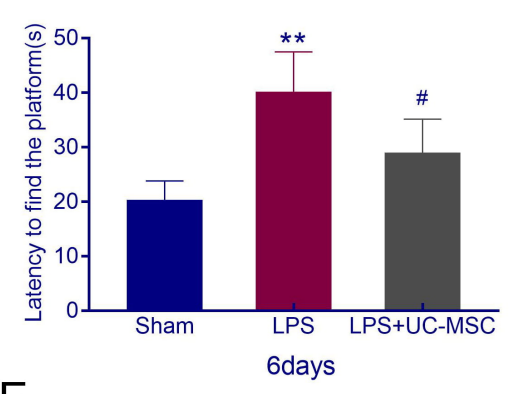

$E$

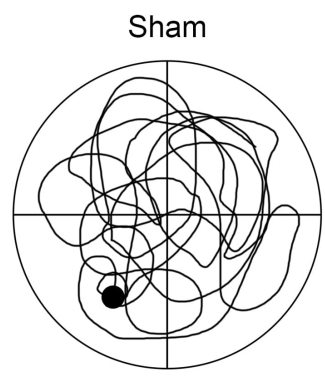

C
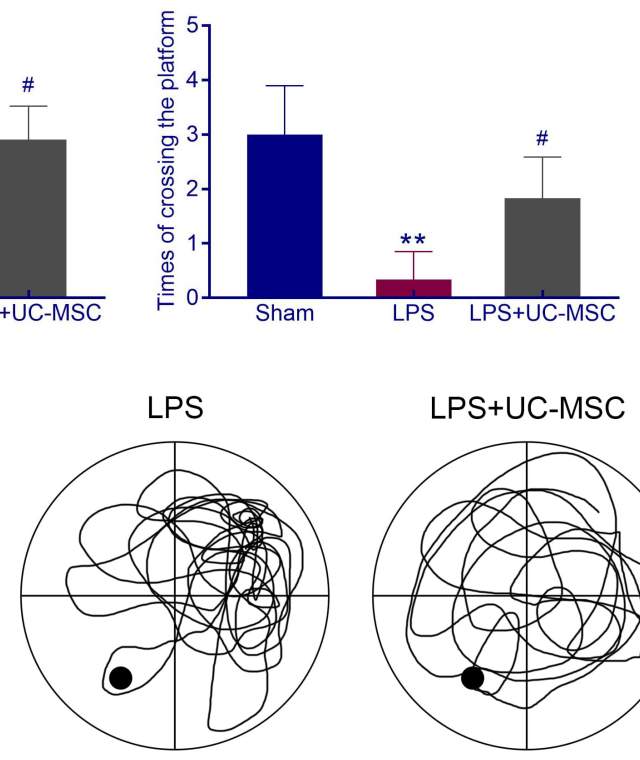

LPS+UC-MSC

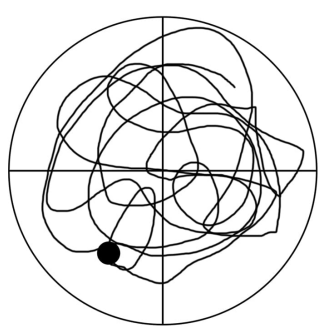

Fig. 3. UC-MSCs alleviated the cognitive dysfunction of SAE mice. (A) The average body weight of mice in sham, LPS, and LPS+ UC-MSCs groups was determined. (B) The latency to find the platform of mice in sham, LPS, and LPS+ UC-MSCs groups were recorded during the Morris water maze test. (C) Times of crossing the platform of mice in sham, LPS, and LPS+ UC-MSCs groups were recorded during Morris water maze test. (D) The swimming speed mice in sham, LPS, and LPS+ UC-MSCs groups were determined during Morris water maze test. (E) The movement routes of mice in sham, LPS, and LPS+ UC-MSCs groups were recorded during the Morris water maze test. ${ }^{* *}: p<0.01$ means significant difference vs. sham group. \#: $p<0.05$ means significant difference vs. LPS group.

\subsection{UC-MSCs inhibited cell apoptosis of cerebral cortex in SAE} mice

Cell apoptosis was remarkably induced by LPS in the cerebral cortex of SAE mice, which was inhibited by UCMSCs treatment $(p<0.01$, Fig. 2A). Furthermore, apoptosisrelated proteins including cleaved caspase- 3 and Bax were increasedly expressed, while Bcl-2 was decreased in SAE mice, which was reversed by UC-MSCs treatment $(p<0.01$, Fig. 2B).

\subsection{UC-MSCs alleviated the cognitive dysfunction of SAE mice}

The average body weight was significantly decreased in SAE mice compared to sham mice $(p<0.01)$, which was restored by UC-MSCs treatment ( $p<0.05$, Fig. 3A). Then, the cognitive dysfunction of SAE mice was explored using the Morris water maze test. Results showed that the latency to find the platform was increased in SAE mice compared to sham mice $(p<0.01)$, while UC-MSCs administration decreased it ( $p<0.05$, Fig. 3B). Besides, times of crossing the platform were decreased in SAE mice compared to sham mice $(p<0.01)$, which was reversed by UC-MSCs treatment ( $p<0.05$, Fig. 3C). Furthermore, the swimming speeds were decreased in SAE mice compared to sham mice $(p<$ $0.01)$, which was improved by UC-MSCs treatment $(p<0.05$, Fig. 3D). The movement route of mice in each group was presented in Fig. 3E.

\subsection{UC-MSCs alleviated the cerebral cortex neuron injury of SAE mice}

The cerebral cortex of sham mice presented a normal histological structure with regular architecture and clear boundary (Fig. 4A). In SAE mice, cerebral cortex neuron injury was observed, characterized by condensed and hyperchromic nuclei, smaller cell bodies with perineuronal vacuolations around the degenerative neurons (Fig. 4A). However, UCMSCs administration improved the abnormal morphology of the cortex and decreased the number of degenerated neurons (Fig. 4A). Moreover, Evans Blue leakage detection experiment was conducted to evaluate BBB integrity. It was observed that Evans Blue leakage was increased in SAE mice ( $p$ $<0.01$ ), which was decreased by UC-MSCs treatment ( $p<$ 0.05, Fig. 4B).

\subsection{UC-MSCs activated PI3K/AKT pathway}

The phosphorylation of PI3K and AKT were decreased in SAE mice ( $p<0.01$, Fig. 5). However, UC-MSCs administration rescued the decrease of phosphorylated PI3K and AKT $(p<0.05$, Fig. 5).

\section{Discussion}

As one of the most common brain diseases in the ICU, SAE presented as a severe diffuse brain dysfunction accompanied by cognitive dysfunction $[1,2]$. At present, controlling inflammation is still the primary treatment for SAE patients, 
A

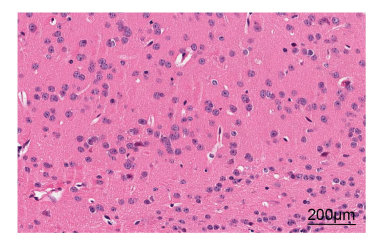

LPS

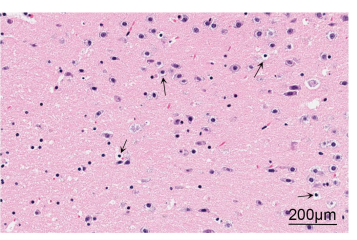

LPS+UC-MSC

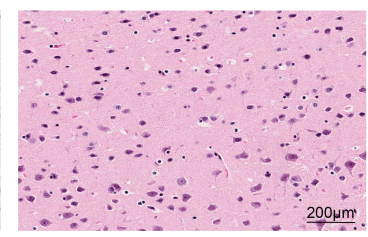

B

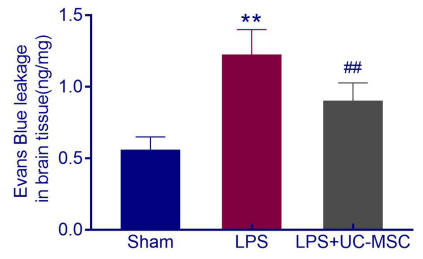

Fig. 4. UC-MSCs alleviated the cerebral cortex neuron injury of SAE mice. (A) HE staining was conducted to assess cerebral cortex neuron injury of UC-MSCs-treated SAE mice. (B) BBB integrity was assessed by Evans Blue leakage detection experiment. The black arrows indicated damaged neurons. **: $p<0.01$ means significant difference vs. sham group. \#\#: $p<0.01$ means significant difference vs. LPS group.
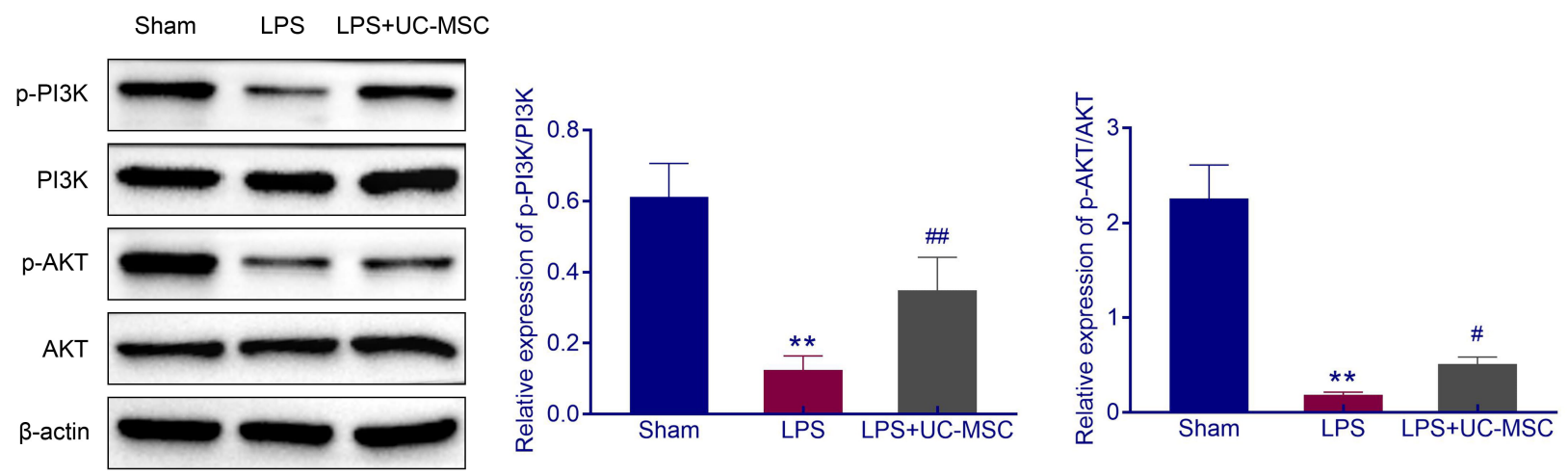

Fig. 5. UC-MSCs activated PI3K/AKT pathway. The protein levels of p-PI3K, p-PI3K, p-AKT, and AKT in brain tissues were detected using Western blot. ${ }^{* *}: p<0.01$ means significant difference vs. sham group. \#: $p<0.05$ means significant difference vs. LPS group. \#\#: $p<0.01$ means significant difference vs. LPS group.

although long-term neurocognitive deficits remain to be resolved [5]. Therefore, it is essential to search for a promising strategy for treating neurocognitive deficits in SAE patients. The protective roles of UC-MSCs in treating diseases including sepsis have been found [12]. However, its role in SAE was unclear. Therefore, this work focused on investigating the effect of UC-MSCs on SAE and uncovering the potential mechanism.

To determine the role of UC-MSCs in SAE, the SAE were constructed in mice. As a component of gram-negative bacteria cell wall, LPS is a mediator of sepsis $[1,5]$. LPS has been extensively used to induce sepsis and its related complications in vitro and in vivo [5]. Therefore, in this work, SAE mice models were established through LPS administration. The pathogenesis of SAE is based on the invasion of bacteria, viruses, or other pathogens in very old cases, young cases, pregnant women, or cases with severe injuries, weakened immune systems, catheters, or a breathing tube. Therefore, neuroinflammation was found in SAE [17] accompanied by increased production of pro-inflammatory factors such as early pro-inflammatory factor IL- 6 , IL- $1 \beta$, TNF- $\alpha$ and late pro-inflammatory factor HMGB1 $[4,17,18]$. Therefore, the productions of inflammatory cytokines in SAE mice were determined. Results revealed that the levels of inflammatory cytokines IL-6, IL- $1 \beta$, TNF- $\alpha$, and HMGB1 in brain tissues of SAE mice were increased. However, the promotional effect of LPS on inflammatory cytokines production in SAE was inhibited by UC-MSCs treatment. Furthermore, considering NF- $\kappa \mathrm{B}$ was a significant mediator of inflammation [19], the expression of phosphorylated NF- $\kappa$ B was determined in SAE mice. Results indicated that expression of phosphorylated NF- $\kappa$ B was increased in brain tissues of SAE mice, which was inhibited by UC-MSCs, suggesting the protective effect of UC-MSCs on inflammation in SAE. Moreover, as is known that neuroinflammation was resulted from microglia activation [20], the expression of microglial activation related biomarker such as Iba1 was detected in SAE mice. It was observed that Iba1 was increased in brain tissues of SAE mice, while was suppressed by UC-MSCs. Therefore, these findings revealed that UC-MSCs inhibited inflammation in SAE. UC-MSCs also exhibited anti-inflammatory effects in other diseases such as type 2 diabetes rats [21] and collagen type II-induced arthritis [22]. Given the role of UC-MSCs in controlling inflammation in SAE, it is reasonable to speculate that UC-MSCs have the potential to treat SAE. 
Previous research revealed that the pathology and histopathology of SAE were mainly involved in the cerebral cortex, while rarely affected the deeper structures and the spinal cord [23, 24]. Thus, the cerebral cortex damage in SAE mice was evaluated. Results showed that cerebral cortex cell apoptosis and neuron injury were observed in SAE mice, consistent with $[25,26]$ results. The previous study revealed that UC-MSCs could inhibit cell apoptosis of injured neurons induced by hypoxic-ischemic injury [27]. UC-MSCs alleviated neurological disorders via suppressing mitogen-activated protein kinase pathway-mediated apoptosis [28]. To further investigate the potential of UC-MSCs in treating SAE, the effect of UC-MSCs in cerebral cortex cell apoptosis and neuron injury was assessed. It was observed that UC-MSCs administration inhibited cell apoptosis and cerebral cortex neuron injury of cerebral cortex in SAE mice.

Cognitive dysfunction was a significant symptom of SAE clinically related to increased mortality [5]. Therefore, the protective effect of UC-MSCs on cognitive dysfunction in SAE mice was explored. Results indicated that the UC-MSCs alleviated the cognitive dysfunction of SAE mice. The beneficial effect of UC-MSCs on improving cognitive dysfunction has been reported previously [29]. Zhou et al. [29] found that UC-MSCs transplantation effectively improved cognitive and neurological function caused by traumatic brain injury. These evidences confirmed the function of UC-MSCs in alleviating cognitive impairment. In addition to controlling inflammation, UC-MSCs also improved cognitive impairment, which may be an advantage for UC-MSCs as a treatment strategy for SAE.

Finally, the potential mechanism of UC-MSCs' protective effect on SAE was explored. The findings revealed that UC-MSCs increased the phosphorylation of PI3K and AKT. In other words, UC-MSCs activated PI3K/AKT pathway. $\mathrm{PI} 3 \mathrm{~K} / \mathrm{AKT}$ pathway has been reported to participate in the SAE development, and the inhibition of this pathway contributed to improving SAE [15, 30]. Tang et al. [30] revealed that Metformin attenuated sepsis-induced brain injury by suppressing oxidative stress, neuroinflammation and apoptosis via regulating the PI3K/AKT pathway. Therefore, these findings suggested that UC-MSCs might protect mice from SAE via activating the PI3K/AKT pathway.

UC-MSCs alleviated inflammation, cell apoptosis and neuron injury of the cerebral cortex, and cognitive dysfunction of SAE, making UC-MSCs therapy a promising therapeutic strategy for SAE treatment.

In this study, the survival time of mice after LPS injection and long-lasting effect of administration of UC-MSCs in SAE model remained elusive. These problems will be furtherly explored in the future study.

\section{Abbreviations}

SAE, Sepsis-associated encephalopathy; UC-MSCs, umbilical cord mesenchymal stem cells; LPS, lipopolysaccharide; ELISA, enzyme-linked immunosorbent assay; BBB, blood- brain barrier; MSCs, mesenchymal stem cells.

\section{Author contributions}

$\mathrm{ZZ}$ and LW designed the study, supervised the data collection, FL analyzed the data, interpreted the data, $X Q, Z H$, LW, YJ and $\mathrm{HH}$ prepare the manuscript for publication and reviewed the draft of the manuscript. All authors have read and approved the manuscript.

\section{Ethics approval and consent to participate}

Ethical approval was obtained from the Ethics Committee of Zhejiang Chinese Medicine University (Approval No. 201809-0290).

\section{Acknowledgment}

Thanks to all the peer reviewers for their opinions and suggestions.

\section{Funding}

This work was supported by the Science and Technology Development Program of Hangzhou City, Zhejiang Province (Grant No. 20201203B56).

\section{Conflict of interest}

The authors declare no conflict of interest.

\section{References}

[1] Gofton TE, Young GB. Sepsis-associated encephalopathy. Nature Reviews Neurology. 2012; 8: 557-566.

[2] Helbing D, Böhm L, Witte OW. Sepsis-associated encephalopathy. Canadian Medical Association Journal. 2018; 190: E1083E1083.

[3] Xu X, Liu L, Wang Y, Wang C, Zheng Q, Liu Q, et al. Caspase-1 inhibitor exerts brain-protective effects against sepsis-associated encephalopathy and cognitive impairments in a mouse model of sepsis. Brain, Behavior, and Immunity. 2019; 80: 859-870.

[4] Tian M, Qingzhen L, Zhiyang Y, Chunlong C, Jiao D, Zhang $\mathrm{L}$, et al. Attractylone attenuates sepsis-associated encephalopathy and cognitive dysfunction by inhibiting microglial activation and neuroinflammation. Journal of Cellular Biochemistry. 2019; 120: 7101-7108.

[5] Kikuchi DS, Campos ACP, Qu H, Forrester SJ, Pagano RL, Lassègue $\mathrm{B}$, et al. Poldip2 mediates blood-brain barrier disruption in a model of sepsis-associated encephalopathy. Journal of Neuroinflammation. 2019; 16: 241.

[6] Yoo J, Kim H, Hwang D. Stem cells as promising therapeutic options for neurological disorders. Journal of Cellular Biochemistry. 2013; 114: 743-753.

[7] Shetty P, Cooper K, Viswanathan C. Comparison of proliferative and multilineage differentiation potentials of cord matrix, cord blood, and bone marrow mesenchymal stem cells. Asian Journal of Transfusion Science. 2010; 4: 14-24.

[8] Semenova E, Grudniak MP, Machaj EK, Bocian K, ChroscinskaKrawczyk M, Trochonowicz M, et al. Mesenchymal Stromal Cells from Different Parts of Umbilical Cord: Approach to Comparison \& Characteristics. Stem Cell Reviews and Reports. 2021. (in press)

[9] Xiang E, Han B, Zhang Q, Rao W, Wang Z, Chang C, et al. Human umbilical cord-derived mesenchymal stem cells prevent the progression of early diabetic nephropathy through inhibiting inflammation and fibrosis. Stem Cell Research \& Therapy. 2020; 11: 336. 
[10] Liu C, Zhang H, Tang X, Feng R, Yao G, Chen W, et al. Mesenchymal Stem Cells Promote the Osteogenesis in Collagen-Induced Arthritic Mice through the Inhibition of TNF- $\alpha$. Stem Cells International. 2018; 2018: 4069032.

[11] Thomi G, Surbek D, Haesler V, Joerger-Messerli M, Schoeberlein A. Exosomes derived from umbilical cord mesenchymal stem cells reduce microglia-mediated neuroinflammation in perinatal brain injury. Stem Cell Research \& Therapy. 2019; 10: 105.

[12] Zhang R, Zhu Y, Li Y, Liu W, Yin L, Yin S, et al. Human umbilical cord mesenchymal stem cell exosomes alleviate sepsis-associated acute kidney injury via regulating microRNA-146b expression. Biotechnology Letters. 2020; 42: 669-679.

[13] He X, Ai S, Guo W, Yang Y, Wang Z, Jiang D, et al. Umbilical cord-derived mesenchymal stem (stromal) cells for treatment of severe sepsis: aphase 1 clinical trial. Translational Research. 2018; 199: 52-61.

[14] Narenji SA, Naghdi N, Azadmanesh K, Edalat R. $3 \alpha$-diol administration decreases hippocampal PKA (II) mRNA expression and impairs Morris water maze performance in adult male rats. Behavioural Brain Research. 2015; 280: 149-159.

[15] Deng S, Ai Y, Gong H, Chen C, Peng Q, Huang L, et al. Neuroglobin Protects Rats from Sepsis-Associated Encephalopathy via a PI3K/Akt/Bax-Dependent Mechanism. Journal of Molecular Neuroscience. 2017; 63: 1-8.

[16] Chen S, Tang C, Ding H, Wang Z, Liu X, Chai Y, et al. Maf1 Ameliorates Sepsis-Associated Encephalopathy by Suppressing the NFkB/NLRP3 Inflammasome Signaling Pathway. Frontiers in Immunology. 2020; 11: 594071.

[17] Yu M, Qin C, Li P, Zhang Y, Wang Y, Zhang J, et al. Hydrogen gas alleviates sepsis-induced neuroinflammation and cognitive impairment through regulation of DNMT1 and DNMT3amediated BDNF promoter IV methylation in mice. International Immunopharmacology. 2021; 95: 107583.

[18] Zhang L, Zhang X, Wu T, Pan X, Wang Z. Isoflurane reduces septic neuron injury by HO-1-mediated abatement of inflammation and apoptosis. Molecular Medicine Reports. 2021; 23: 155.

[19] Kunnumakkara AB, Shabnam B, Girisa S, Harsha C, Banik K, Devi $\mathrm{TB}$, et al. Inflammation, NF- $\times \mathrm{B}$, and Chronic Diseases: how are they Linked? Critical Reviews in Immunology. 2020; 40: 1-39.

[20] Streit WJ, Mrak RE, Griffin WS. Microglia and neuroinflamma- tion: a pathological perspective. Journal of Neuroinflammation. 2004; 1: 14.

[21] Sun X, Hao H, Han Q, Song X, Liu J, Dong L, et al. Human umbilical cord-derived mesenchymal stem cells ameliorate insulin resistance by suppressing NLRP3 inflammasome-mediated inflammation in type 2 diabetes rats. Stem Cell Research \& Therapy. 2017; 8: 241 .

[22] Lin CM, Gu J, Zhang Y, Shen LJ, Ma L, Ni J, et al. Effect of UCMSCs on inflammation and thrombosis of the rats with collagen type II induced arthritis. Chinese Journal of Hematology. 2012; 33: 215-219. (in Chinses)

[23] Fülesdi B, Molnar L, Németh N, Molnár C. Sepsis-associated encephalopathy: a review of literature. Neurology India. 2018; 66: 352-361.

[24] Jackson AC, Gilbert JJ, Young GB, Bolton CF. The Encephalopathy of Sepsis. Canadian Journal of Neurological Sciences. 1985; 12: 303-307.

[25] Arslan D, Ekinci A, Arici A, Bozdemir E, Akil E, Ozdemir HH. Effects of Ecballium elaterium on brain in a rat model of sepsisassociated encephalopathy. Libyan Journal of Medicine. 2017; 12: 1369834.

[26] Xie K, Zhang Y, Wang Y, Meng X, Wang Y, Yu Y, et al. Hydrogen attenuates sepsis-associated encephalopathy by NRF2 mediated NLRP3 pathway inactivation. Inflammation Research. 2020; 69: 697-710.

[27] Li F, Zhang K, Liu H, Yang T, Xiao D, Wang Y. The neuroprotective effect of mesenchymal stem cells is mediated through inhibition of apoptosis in hypoxic ischemic injury. World Journal of Pediatrics. 2020; 16: 193-200.

[28] Zhang R, Chen H, Zheng Z, Liu Q, Xu L. Umbilical cord-derived mesenchymal stem cell therapy for neurological disorders via inhibition of mitogen-activated protein kinase pathway-mediated apoptosis. Molecular Medicine Reports. 2015; 11: 1807-1812.

[29] Zhou H, Liu Z, Liu X, Chen Q. Umbilical cord-derived mesenchymal stem cell transplantation combined with hyperbaric oxygen treatment for repair of traumatic brain injury. Neural Regeneration Research. 2016; 11: 107-113.

[30] Tang G, Yang H, Chen J, Shi M, Ge L, Ge X, et al. Metformin ameliorates sepsis-induced brain injury by inhibiting apoptosis, oxidative stress and neuroinflammation via the PI3K/Akt signaling pathway. Oncotarget. 2017; 8: 97977-97989. 